\title{
How we evaluate your manuscripts
}

\author{
Journals differ in how they evaluate submissions, depending on their aims and scope. Here we share how the \\ Nature Human Behaviour editorial team evaluates research manuscripts submitted to the journal.
}

L ike all Nature-branded journals, Nature Human Behaviour does not have an external editorial board-all decisions are made by a team of in-house editors. We are all $\mathrm{PhD}$ holders, passionate about science, and as a team, we evaluate dozens of manuscripts every week.

When we evaluate research manuscripts, we read the manuscript itself as well as the relevant prior literature. Our decisions are not affected by how well-established the authors may be or in which country they work. Instead, we use a number of researchspecific criteria to determine suitability of the work for the journal (see Fig. 1).

A core aspect of our decision-making is based on evaluating the scope of the work, the importance of the research question, and the contribution the manuscript makes to the literature. If the work is likely to be of interest only to other specialists working on the same question, it will be more suitable for a discipline-specific journal. When evaluating how important the research question is, we recognize that there are many ways to define importance. We examine whether the research question is a central yet unanswered or debated one in a specific discipline, whether the work is of interdisciplinary significance (i.e., pursued from different angles by several disciplines), or whether it is a question with immediate practical implications (for instance, could the work directly influence policy or clinical practice?).

We strongly believe that if the research question is important and the methods are robust, the results are also important, no matter what they are. For this reason, we publish studies with null results, as well as Registered Reports.

We believe that scientific advances take many shapes and we look for manuscripts that represent an advance in at least one of four categories (Fig. 1). Manuscripts may represent an advance for the field by being conceptually novel or methodologically novel. However, we consider evidencebased advances to be equally important: a manuscript may lack conceptual or methodological novelty, but may be of unprecedented scale or rigour, providing a definitive answer to a debated research question. Rigorous replication studies of highly influential but debated prior research belong to this category. We also prioritize for

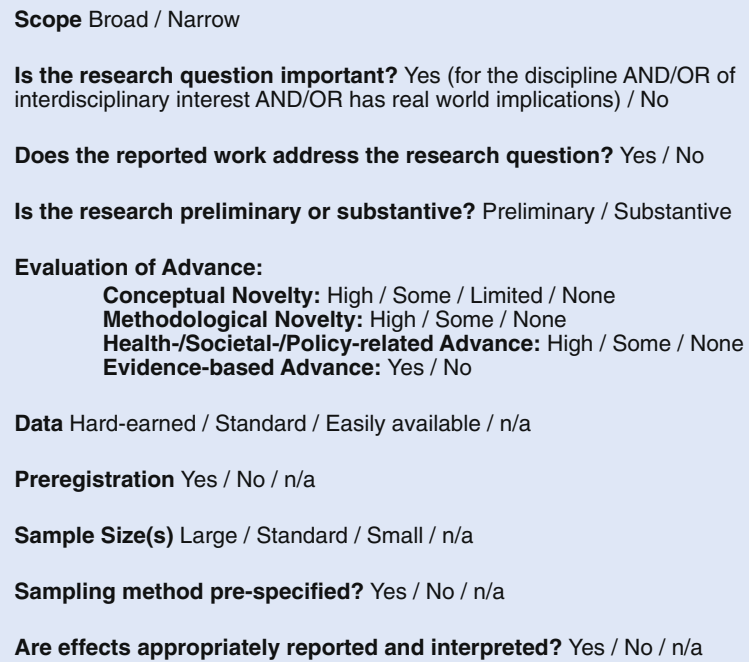

Fig. 1 | The criteria the Nature Human Behaviour editors use to evaluate initial research submissions.

publication studies that represent a health- or policy-related advance, even if they are not conceptually novel. Studies in this category include work that takes a research question out of the lab and into the field.

We look for work that is thorough, addresses the research question, and provides substantial evidence to support its conclusions. If we feel that the work is preliminary but promising, we may make suggestions on what types of evidence could strengthen the work for resubmission.

For empirical work, we prioritize for peer review manuscripts that report hard-earned data, studies that were pre-registered, studies that are highly powered, and those for which the sampling method was specified in advance. Our evaluations, however, do not focus in detail on the methods-we rely on our reviewers for this.

We welcome presubmission enquiries, and these can be a quick way of finding out whether your work is within the scope of the journal and if it is on a topic that would appeal to a broad cross-section of our audience. However, our responses to presubmission enquiries are necessarily tentative. If your manuscript is ready, your best bet is to go ahead and submit it-that's the quickest way to have a definite decision.
For manuscripts that we send out to review, we value reviewer suggestions. However, our reviewer selection always also includes experts who were not suggested by the authors. We do our best to ensure that our reviewers are independent by checking co-publication records and institutional affiliations, but also ask our reviewers to contact us if they feel they have a conflict. We invariably respect reviewer exclusions and will not invite reviewers you have indicated might be biased. If your exclusions are extensive and make it difficult for us to identify suitably qualified reviewers, we will contact you to ask for a more circumscribed set of up to four individuals.

If we decide not to peer review your manuscript, our decision letter will include the key reason or reasons for our decision, for instance, strength of advance over the existing literature, strength of evidence in support of the manuscript's conclusions, breadth of scope, or suitability of the evidence to answer the research question (for example, if the question is causal but the evidence correlational).

Over the past 12 months, we have sent out for review $19 \%$ of the manuscripts we received. For work we do not take forward to peer review, we will frequently make suggestions for other journals within the 
Nature Research group that may be a better fit for your work. If you have indicated that you are happy for us to do so, we may consult with the editors of other Nature Research journals to see if they would commit to peer reviewing your work

Regardless of outcome, we feel privileged to have the chance to read some of the most interesting new work across the numerous disciplines we cover. We try hard to make decisions consistently and we discuss manuscripts extensively as a team when a decision isn't straightforward. If you feel our evaluation may have missed important aspects of your work, you are welcome to appeal the decision, providing further explanation.
Still unclear on how we make decisions on initial research submissions? Do you have any comments or suggestions? We welcome your feedback at humanbehaviour@nature.com.

Published online: 13 November 2019 https://doi.org/10.1038/s41562-019-0778-0 